\title{
Automatic determination of source parameters of the 2007 Noto Hanto earthquake
}

\author{
Changjiang Wu and Shigeki Horiuchi \\ National Research Institute for Earth Science and Disaster Prevention, 3-1 Tennodai, Tsukuba, Ibaraki 305-0006, Japan
}

(Received June 29, 2007; Revised November 21, 2007; Accepted December 5, 2007; Online published November 7, 2008)

\begin{abstract}
Large earthquakes have been observed to be caused by the rupture of several asperities. The rapid determination of source parameters is hence of great importance for correctly determining shaking intensities or evaluating the strong motion distribution generated by a large earthquake. This study shows that we can automatically preprocess strong motion data using automatic $P$ - and $S$-phase pickers, determine the focal mechanism, and identify the fault plane from two nodal planes. A real-time simulation is performed for the 2007 Noto Hanto earthquake. Using our automatic fault plane identificator, we were able to identify one of the nodal planes with a strike of $62^{\circ}$ and a dip of $70^{\circ}$ within a 30-s interval from the beginning of focal mechanism determination to the end of waveform inversion. Results from automatic inversions showed that the slip was confined to a shallow portion with a maximum slip of about $3 \mathrm{~m}$. The total seismic moment released in $10 \mathrm{~s}$ was $2.0 \times 10^{18} \mathrm{~N} \mathrm{~m}$, which corresponds to a moment magnitude of 6.8.
\end{abstract}

Key words: Real-time, earthquake, source parameter, rupture process.

\section{Introduction}

With the enhancement of regional seismic networks as well as the rapid development of internet communication technology and computer technology, real-time seismology has become a practical approach to effectively mitigate damage from earthquakes. For example, using $P$-wave arrival times and amplitudes from only a few stations, a realtime earthquake analysis system developed by Horiuchi et al. (2005) can precisely locate the earthquake within a few seconds and issue an early-warning for the $S$-wave shaking. This system is installed in the National Research Institute for Earth Science and Disaster Prevention (NIED) and worked well when the Noto Hanto earthquake occurred on May 25, 2007. This automatic procedure, however, models an earthquake as a point source, which provides insufficient data for determining shaking intensity or evaluating strong motion distribution caused by a large earthquake, since large earthquakes are usually observed as twodimensional asperities (e.g., Yamanaka and Kikuchi, 2004).

Several methods aimed at rapidly and automatically determining earthquake fault planes or revealing the rupture process have been proposed (e.g., Dreger and Kaverina, 2000; Kuge, 2003; Hori, 2004). After the moment of tensor inversion, one should determine which of the nodal planes of the focal mechanism is the fault plane and subsequently estimate the dimensions of the faulting area before the finite-plane source inversion. A finite-fault model was thus obtained in 11-20 min by applying Dreger and Kaverina's method for the earthquakes with $M>5.5$ in Northern California. Kuge (2003) proposed a similar automatic

Copyright (c) The Society of Geomagnetism and Earth, Planetary and Space Sciences (SGEPSS); The Seismological Society of Japan; The Volcanological Society of Japan; The Geodetic Society of Japan; The Japanese Society for Planetary Sciences; TERRAPUB method and verified it using simulations of six earthquakes in Japan. In contrast, Hori (2004) proposed a method for rapidly estimating the seismic fault plane using the aftershock hypocenters in a short period. Unfortunately, however, all of these methods are time consuming and impractical for a real-time warning system. In this study, we proposed an automatic method leading toward real-time determination of the finite-fault slip distribution.

\section{Methodology}

Our methodology is shown in as a flow chart in Fig. 1. Instead of waiting for the moment tensor solutions of a larger earthquake, we determine the focal mechanism using the amplitude (with polarity) of the $P$-wave first motions as soon as $P$-wave arrivals are detected. The focal mechanism consists of two nodal planes, and the fault plane is identified by comparing the moment indexes of the two nodal planes using $P$-waves only. Given that the magnitude of a larger earthquake is known (it has been reliably estimated by the Horiuchi system; Yamamoto et al., 2007), waveform inversion for a finite-fault plane source model is then carried out.

\subsection{Automatic $\boldsymbol{P}$-wave and $S$-wave pickers}

Robust and automatic $P$-wave detection is of crucial importance for a real-time earthquake location system. $P$ wave particle motion is usually observed in a direction that is nearly perpendicular to the surface, whereas particle motion of noise is random and surface-wave-like. It is this property of $P$-waves, in addition to the ratios of the short time average (STA) to the long time average (LTA), which is used for our $P$-wave picker. To estimate the magnitude and to identify the fault plane from the $P$-wave, we also need an automatic $S$-wave picker. Our $S$-wave picker takes into account the physical property that the plane of $S$-wave particle motion is perpendicular to the direction of the $P$-wave 


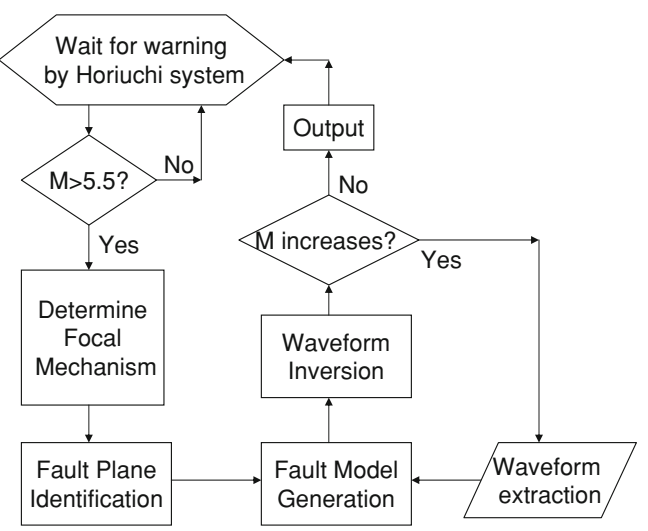

Fig. 1. Flow chart of the automatic inversion method. Simulation is performed given that the warning of an earthquake with $M=6.0$ is issued.

particle motion. This property has been widely used for automatic detection of $S$-waves (e.g., Cichowicz, 1993; Oonincx, 1999). Following the denotation of Oonincx, we constructed a cross-power matrix $\left\{C_{i j}\right\}$ of a three-component seismic signal $u=\left(u_{1}, u_{2}, u_{3}\right)$

$$
C_{i j}=\frac{1}{N} \sum_{k=i}^{i+N-1} u_{i}(k) u_{j}(k)
$$

and calculated four characteristic functions as follows

$$
\begin{aligned}
\kappa_{p} & =\frac{\lambda_{1}-\lambda_{2}}{\lambda_{1}+a} \\
\kappa_{1} & =\frac{2}{\pi} \arccos \left(\frac{\left|v_{1} \cdot v_{p}\right|}{\left\|v_{1}\right\| \cdot\left\|v_{p}\right\|}\right) \\
\kappa_{2} & =\frac{\left(\lambda_{1}-\lambda_{3}\right)^{2}+\left(\lambda_{2}-\lambda_{3}\right)^{2}}{\lambda_{1}^{2}+\lambda_{2}^{2}+\lambda_{3}^{2}+a^{2}} \\
\kappa_{3} & =1-\frac{\sum\left\|v_{p} \cdot u\right\|^{2}}{\sum\|u\|^{2}}
\end{aligned}
$$

where $\lambda_{i}$ are the eigenvalues of the cross matrix $\boldsymbol{C}$ with $\lambda_{1} \geq \lambda_{2} \geq \lambda_{3}, v_{i}$ is the corresponding eigenvectors, $v_{p}$ is the vector of the $P$-wave direction, and $a$ is an adjusting parameter equal to the largest eigenvalue of the background noise cross power matrix. Here we have a robust estimator for $P$-wave arrivals

$$
T_{P}=T\left(\max (\mathrm{STA} / \mathrm{LTA}) \mid \kappa_{P} \geq 0.8\right)
$$

and for $S$-wave arrivals

$$
T_{S}=T\left(\max \left(\kappa=\kappa_{1}^{2} \cdot \kappa_{2}^{2} \cdot \kappa_{3}^{2}\right) \mid \mathrm{STA} / \mathrm{LTA} \geq 2\right)
$$

where $\max (x)$ means a local maximum of $x$.

\subsection{Fault plane identificator}

In order to rapidly identify the fault plane from the two nodal planes of the focal mechanism, the Green's functions $G_{n}(t ; \tau, i, j)$ used in this step are simplified so that only the geometrical spreading effects and radiation effects (Aki and Richards, 1980) are considered. The released moment $m(\tau, i, j)$ at the time step $\tau$ at the $(i, j)$ grid can be determined by minimizing the error between

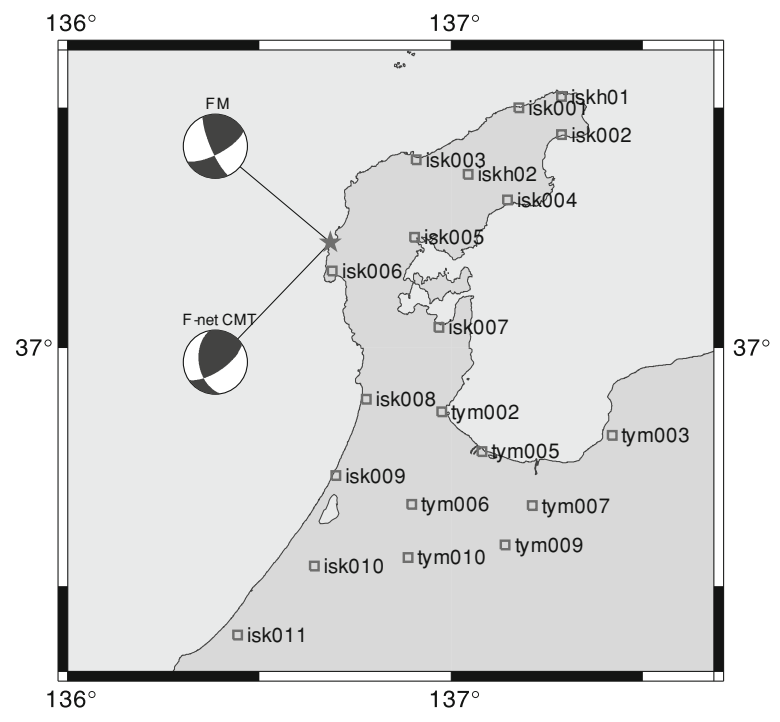

Fig. 2. Station map. Squares mean the location of stations used in this study. The epicenter is marked by the star. Two mechanisms listed in Table 1 solutions are shown, where FM means the focal mechanism solution obtained in this study.

the observed amplitude $u_{n}(t)$ and the predicted amplitude $m(\tau ; i, j) \cdot G_{n}(t ; \tau, i, j)$. For simplicity, only grids inside a ring between $r$ (in $\mathrm{km}$ ) $=2.0 t$ and $r=3.5 t$ (in second) are searched at each time step. The moment release at each grid can be estimated as follows

$$
m(\tau, i, j)=\frac{\sum u_{n}(t) G_{n}(t ; \tau, i, j)}{\sum G_{n}^{2}(t ; \tau, i, j)}
$$

If one summarizes the released moment of all grids at all time steps for the two nodal planes, respectively, the sum on the actual rupture plane should be smaller than the other one. Summation of $m(\tau, i, j)$ is hence defined as the fault plane identificator. It corresponds to a minimum moment inversion (Ji et al., 2002).

\subsection{Automatic waveform inversion}

To rapidly invert the source process, one should first construct a database of standard Green's functions. The standard Green's function can be readily convolved with any source time function and interpolated at any distance. In this study, the Green's functions were calculated using the reflection/transmission matrix method (Kennett and Kerry, 1979). Using the magnitude $M$ issued by Horiuchi system, we estimate the fault size as follows

$$
L=10^{0.6 * M-2}
$$

The fault plane is divided into small subfaults, allowing their slip vectors to be rotated up to $90^{\circ}$. The source time function on each subfault is expressed by six halfoverlapped isosceles triangles with a width of $1 \mathrm{~s}$. The inversion method has been described in detail in previous studies (see, for example, Wu and Takeo, 2004).

\section{Strong Motion Data and Preparation for Inver- sion}

Strong motion data recorded at $18 \mathrm{~K}-\mathrm{NET}$ stations and two KiK-net stations were used for simulation (Fig. 2). $P$ - 


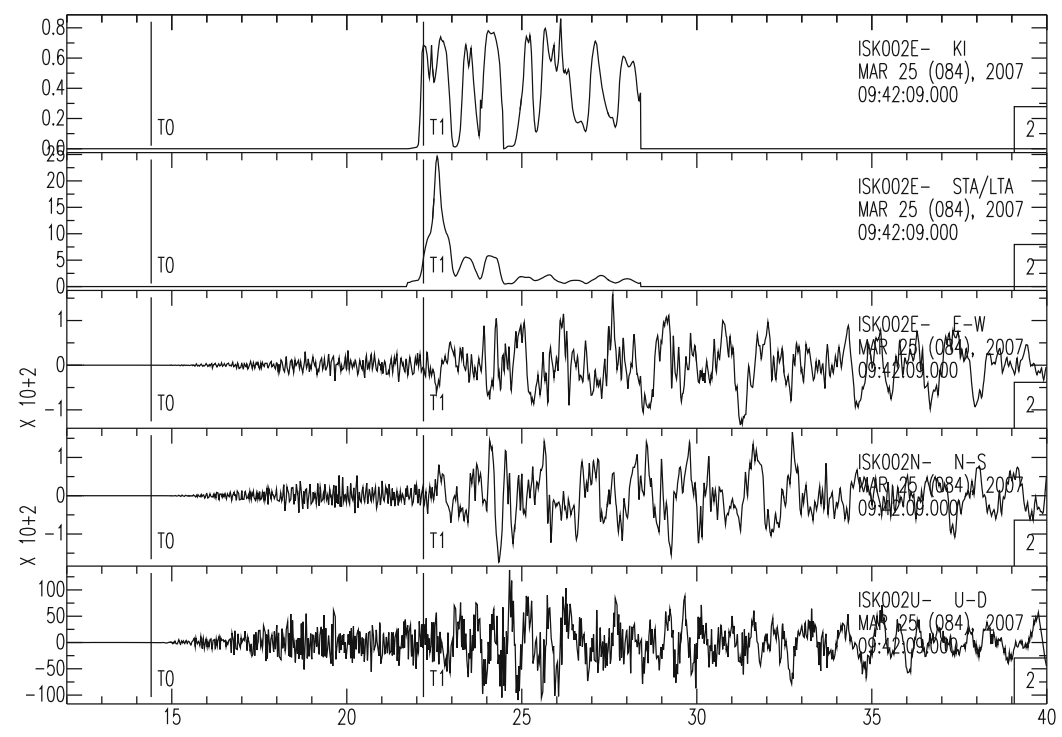

Fig. 3. Example of automatic $S$-phase picker for records. T0 is detected as the $P$-phase arrival and T1 as the $S$-phase arrival. The upper two show calculated indices (see formula (7)) for $S$-wave picker. The lower three show the seismogram recorded at ISK002.

Table 1. Focal mechanism and fault plane identification.

\begin{tabular}{crrrc}
\hline Nodal & Strike & Dip & \multicolumn{1}{c}{ Rake } & Identification Index \\
\hline 1 & 62.01 & 69.53 & 167.16 & 118.46 (identified) \\
2 & 156.56 & 77.98 & 20.95 & 334.89 (rejected) \\
F-net MT & $56 / 173$ & $66 / 48$ & $132 / 34$ & \\
\hline
\end{tabular}

and $S$-wave arrival times were picked automatically by the $P$ - and $S$-wave pickers. An example is shown in Fig. 3. Table 1 lists the focal mechanism as well as the identification index of the fault plane. The F-net Moment tensor solution is also listed for comparison. The velocity model proposed by Ukawa et al. (1984) was used for calculating Green's functions.

\section{Simulation Results}

An automatic source inversion was simulated for the 2007 Noto Hanto earthquake in the same computer where the Horiuchi system is operated. It took less than $15 \mathrm{~s}$ to identify the fault plane using $P$-waves at 20 stations. The fault plane is automatically divided into $7 \times 6$ subfaults (of size $4 \times 4 \mathrm{~km}$ ). Approximately another $15 \mathrm{~s}$ is needed to invert the source process if we use $5 \mathrm{~Hz}$-sampled waveforms with a duration of $30 \mathrm{~s}$ recorded at eight stations (automatically selected according the order of $P$-wave arrival times). As Fig. 4 shows, the large slip is confined to the shallow part around the hypocenter, with the maximum slip of about $3 \mathrm{~m}$. The seismic moment determined from this finite source model is $2.0 \times 10^{18} \mathrm{~N} \mathrm{~m}$, corresponding to a moment magnitude of 6.8. The waveform fitting is excellent considering that only eight stations are included (see Fig. 5). Our result is consistent with the source model inverted from teleseismic body waves (Yamanaka, 2007).

\section{Discussion and Conclusions}

Using a novel approach to quickly determine the fault plane, we are able to determine the focal mechanism based

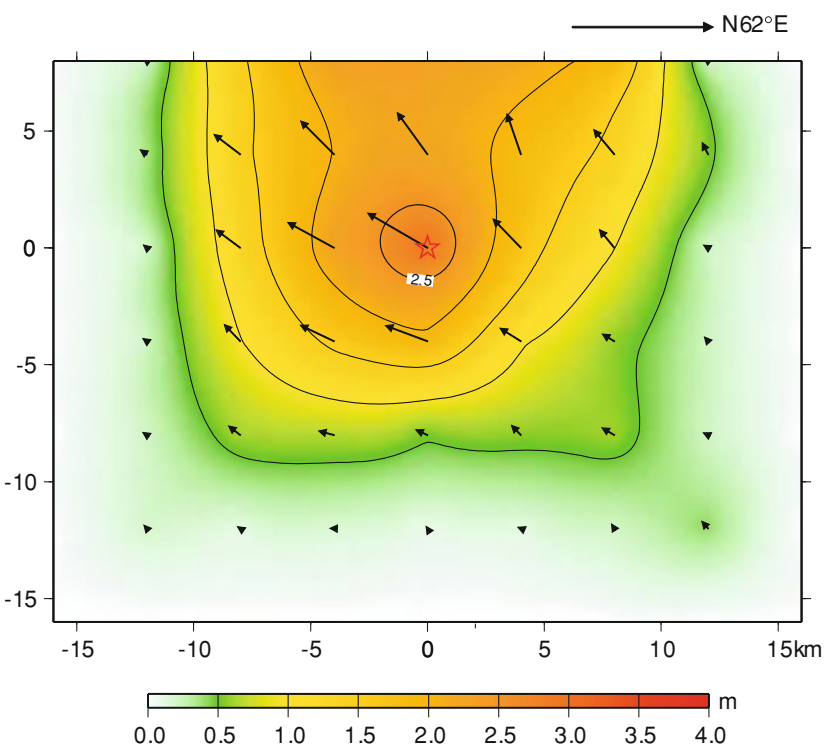

Fig. 4. Slip and slip vector distribution of the Noto Hanto earthquake obtained from the automatic inversion using records at 8 stations. Hypocenter is marked by a red star. The flame axes are given in kilometers.

on the amplitude (with polarity) of $P$-wave first motions. A proper velocity model is required for a stable solution of the focal mechanism. The automatic procedure needs to wait for CMT solutions when the focal mechanism solution is not satisfactory. Once the focal mechanism is determined, the more observations that are used, the more reliable the identification of the fault plane becomes. To achieve this, a natural feature of a real-time system can be used: the length of the signals and the number of available stations increase with time.

Inversion results can also be updated in a real-time sense. Figure 6 shows the snapshots of slip distribution using strong motion data at eight stations initially, then at four more stations that were added for each snapshot. It is inter- 


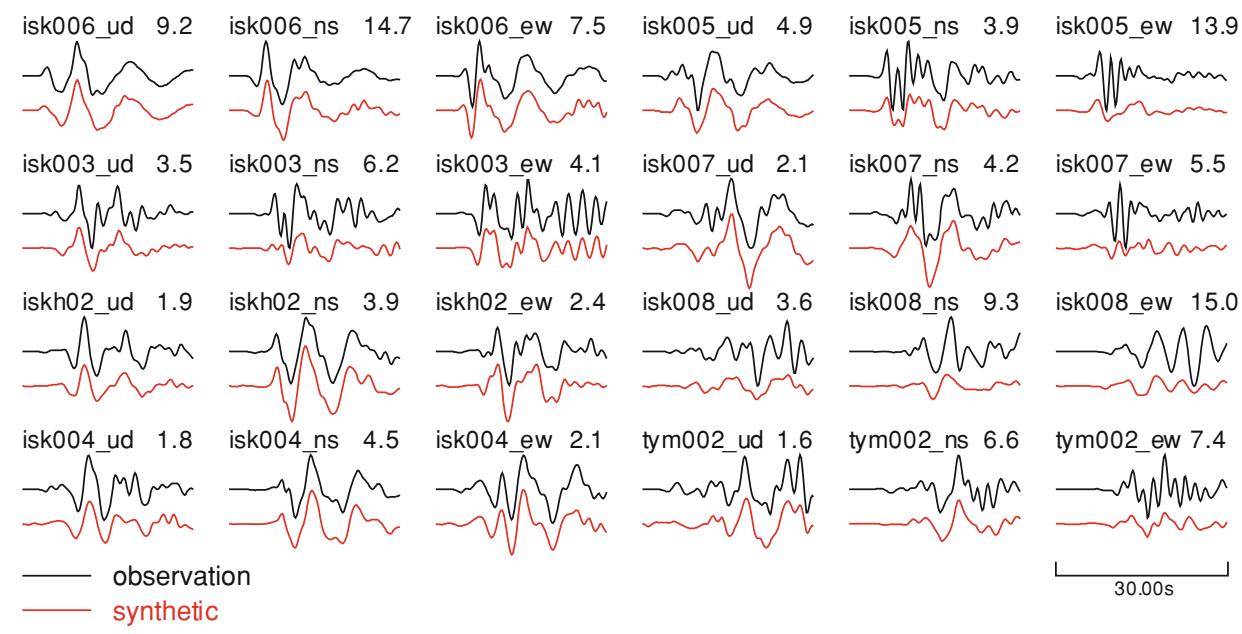

Fig. 5. Comparison of observation waveform (black) with synthetic waveform (red). The maximum amplitude (in $\mathrm{cm} / \mathrm{s}$ ) is shown on the upper right corner for each component.
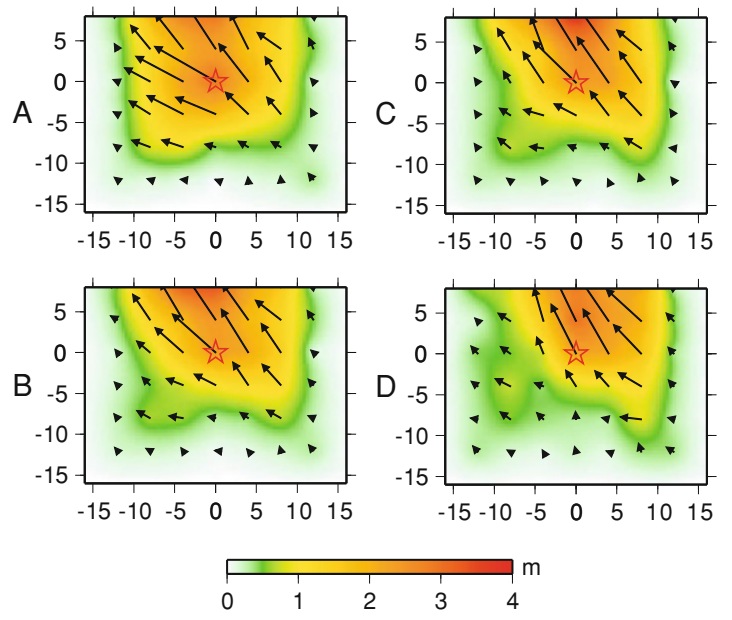

Fig. 6. Comparison of slip distributions of the Noto Hanto earthquake using different sets of records. A: slip inverted from records of eight stations; B: slip inverted from 12 stations; C: slip inverted from 16 stations; D: slip inverted from 20 stations. The flame axes are given in kilometers.

esting to note that the asperity area (i.e. large slip) moved landward when more stations were included while at the same time the time consumed for Green's function convolution and waveform inversion increased to $30 \mathrm{~s}$. Because of the trade-off between the resolution and time, automatic selection of stations on the basis of station azimuth should be considered when more and more stations are available for inversion. In the case of a large earthquake, such a selection could save significant time.

We should point out that our simulation was performed using offline K-NET and KiK-net strong motion data. We will test this method for other large earthquakes using $\mathrm{K}$ NET or KiK-net strong motion data, but it is still not a practical real-time procedure unless we can successfully perform simulations using real-time Hi-net data. Since Hinet sensors have a much narrower dynamic range and show a cutoff of frequencies below $1 \mathrm{~Hz}$ (Obara, 2005), real-time source process inversion will become more difficult.
In conclusion, using our automatic $P$-wave and $S$-wave pickers, we can read the amplitude (polarities included) of $P$-wave first motions and determine the focal mechanism automatically. The fault plane can be identified from the two nodal planes using the $P$-phase data. It takes $30 \mathrm{~s}$ for an automatic inversion when there are less than ten stations. When we performed a simulation for the 2007 Noto Hanto earthquake, we were able to identify one of the nodal planes with a strike of $62^{\circ}$ and a dip of $70^{\circ}$ as the fault plane using our automatic fault plane identification method. The result from automatic inversion showed that the slip was confined to shallow part with the maximum slip of about $3 \mathrm{~m}$. The total seismic moment released in $10 \mathrm{~s}$ was $2.0 \times 10^{18} \mathrm{~N} \mathrm{~m}$, corresponding to a moment magnitude of 6.8 .

Acknowledgments. This study is supported by the leading project 'Research Project for the Practical Use of Real-time Earthquake Information networks'. We used strong-motion seismograms recorded by the K-NET and KiK-net of NIED, Japan.

\section{References}

Aki, K. and P. G. Richards, Quantitative seismology, Freeman and Company, San Francisco, 1980.

Cichowicz, A., An automatic S-phase picker, Bull. Seismol. Soc. Am., 83, 180-189, 1993.

Dreger, D. S. and A. Kaverina, Seismic remote sensing for the earthquake source process and near-source strong shaking: a case study of the October 16, 1999 Hector Mine earthquake, Geophys. Res. Lett., 27, 1941-1944, 2000.

Hori, S., Automated methods for rapid estimation of a seismic fault plane, Research Report of National Research Institute for Earth Science and Disaster Prevention, No. 65, 1-23, 2004.

Horiuchi, S., H. Negishi, K. Abe, A. Kamimura, and Y. Fujinawa, An automatic process system for broadcasting earthquake alarms, Bull. Seismol. Soc. Am., 95, 708-718, 2005.

Ji, C., D. J. Wald, and D. V. Helmberger, Source Description of the 1999 Hector Mine, California, Earthquake, Part II: Complexity of Slip History, Bull. Seismol. Soc. Am., 92, 1208-1226, 2002.

Kennett, L. N. and N. J. Kerry, Seismic waves in a stratified half space, Geophys. J. R. Astr. Soc., 57, 557-583, 1979.

Kuge, K., Source modeling using strong-motion waveforms: toward automated determination of earthquake fault planes and moment-release distribution, Bull. Seismol. Soc. Am., 93, 639-654, 2003.

Obara, K., K. Kasahara, S. Hori, and Y. Okada, A densely distributed highsensitivity seismograph network in Japan: Hi-net by National Research Institute for Earth Science and Disaster Prevention, Rev. Sci. Instrum., 
76, 021301-1-12, 2005.

Oonincx, P. J., A wavelet method for detecting $S$-wave in seismic data, Computational Geoscience, 3, 113-134, 1999.

Ukawa, M., M. Ishida, S. Matsumura, and K. Kasahara, Hypocenter determination method of the Kanto-Tokai observational network for microearthquakes, Rep. Natl. Res. Inst. Earth Sci. Disas. Prev., 53, 1-88, 1984.

Wu, C. and M. Takeo, An intermediate deep earthquake rupturing on a dip-bending fault: Waveform analysis of the 2003 Miyagi-ken Oki earthquake, Geophys. Res. Lett., 31, doi:10.1029/2004GL021228, 2004. Yamamoto, S., S. Horiuchi, H. Nakamura, and C. Wu, Effectiveness of seismic intensity magnitude for earthquake early warning, Geophys. Explora., 2007 (in Japanese) (in press).

Yamanaka, Y., The March 25, 2007, Noto Hanto earthquake, EIC Seismol. Note, No. 185, 2007.

Yamanaka, Y. and M. Kikuchi, Asperity map along the subduction zone in northeastern Japan inferred from regional seismic data, J. Geophys. Res., 109, doi:10.1029/2003JB002683, 2004.

C. Wu (e-mail: wu@bosai.go.jp) and S. Horiuchi 